\title{
O uso da biópsia aspirativa com agulha fina (BAAF) no diagnóstico de lesão iriana suspeita - relato de caso
}

\author{
Fine needle aspiration biopsy (FNAB) in the diagnosis of a suspicious iris \\ lesion - case report
}

\author{
Zélia Maria da Silva Corrêa ${ }^{1}$ \\ Ítalo Mundialino Marcon ${ }^{2}$
}

Este trabalho foi realizado no Departamento de Oftalmologia da Santa Casa de Misericórdia de Porto Alegre e foi parcialmente apresentado como Poster no Simpósio Internacional da Santa Casa de São Paulo- Julho-1999.

${ }^{1}$ Chefe do Setor de Oncologia, Serviço de Oftalmologia da Santa Casa de Misericórdia de Porto Alegre (ISCMPA) - RS. Pós-graduanda da Universidade Federal de São Paulo / Escola Paulista de Medicina.

2 Chefe do Serviço de Oftalmologia da Santa Casa de Porto Alegre (ISCMPA) - RS. Mestre e Doutor em Oftalmologia pela Universidade Federal de São Paulo / Escola Paulista de Medicina. Professor Regente da Disciplina de Oftalmologia da Fundação Faculdade Federal de Ciências Médicas de Porto Alegre - FFFCMPA, Porto Alegre - RS.

Endereço para correspondência: Zélia M. Corrêa Av. Nilo Peçanha, 2421 - Porto Alegre (RS) Brasil. E-mail: zmcorrea@terra.com.br

\section{RESUMO}

Relatar o caso de um paciente que apresentou massa intra-ocular sólida no segmento anterior do olho direito e a investigação diagnóstica escolhida pelos autores. A dúvida diagnóstica e a recusa do paciente em aceitar o tratamento levaram os autores a realizar uma biópsia aspirativa com agulha fina da lesão. A biópsia foi realizada por meio de punção por via corneana, através do humor aquoso até a lesão tumoral. A citologia do material coletado na biópsia determinou a estratégia terapêutica neste caso. O diagnóstico citológico foi melanoma maligno primário da coróide do tipo misto. Sugeriu-se a enucleação do globo ocular devido à localização do tumor e seu potencial para metástases à distância. Após a enucleação, o exame anatomopatológico apresentou resultado concordante com a citologia. O paciente está sendo acompanhado clinicamente, sem sinais de metástases 2 anos após enucleação.

Descritores: Neoplasias da coróide/patologia; Melanoma/patologia; Biopsia por agulha; Enucleação ocular; Relato de caso

\section{INTRODUCÃ̃O}

A biópsia aspirativa começou a ser usada na medicina nos anos 30 pelos cirurgiões Martin e Ellis do Hospital Presbiteriano de Nova York ${ }^{(1)}$. Entretanto as técnicas de aspiração só se tornaram populares nos anos 60 através das publicações do Instituto Karolinska na Suécia ${ }^{(2)}$. O primeiro artigo sobre biópsia aspirativa em oftalmologia foi publicado por Veasey em 1951 e o tumor em questão era um sarcoma ${ }^{(3)}$. Vários outros artigos sobre biópsia em oftalmologia foram publicados desde então, entretanto nenhum deles apresentava casuística e tempo de seguimento suficientes para consagrar esta modalidade diagnóstica ${ }^{(4-8)}$.

Recentemente a biópsia aspirativa tem sido estudada com o objetivo de: 1 - elucidar casos suspeitos, onde todos os outros recursos se mostraram inconclusivos, 2 - correlacionar o tipo tumoral (celular) com outros focos tumorais (metastáticos), e 3 - confirmar o diagnóstico clínico quando há recusa ou dúvida quanto ao tratamento por parte do paciente ${ }^{(4-6)}$.

A complexidade técnica, curva de aprendizado, e temores acerca de disseminação tumoral têm impedido o uso mais freqüente deste método no diagnóstico de lesões intra-oculares suspeitas de malignidade ${ }^{(5-6)}$. Além disto, amostras de quantidade insuficiente implicam em decisões complexas, influenciadas pela responsabilidade frente à repetida inconclusão diagnóstica ${ }^{(7)}$. Entretanto deve-se lembrar que o diagnóstico de tumores intraoculares pequenos e suspeitos de malignidade muitas vezes requer confirmação citológica para a indicação de tratamento precoce ${ }^{(8)}$. 


\section{MÉTODOS}

Os autores relatam o caso de um homem branco, com 47 anos, que se apresentou com queixa de baixa da acuidade visual por massa intra-ocular em íris OD invadindo o eixo visual (Figura 1) que foi percebida há 8 meses. Entretanto a perda visual foi notada há 4 meses. O paciente negou quaisquer sintomas sistêmicos durante o último ano. A história pregressa de doenças sistêmicas, trauma ocular e neoplasia, bem como os antecedentes familiares de neoplasia eram negativos. O paciente já havia procurado outro oftalmologista que o orientou a não tratar a lesão. Entretanto, devido à baixa da visão e o aspecto visível da lesão, o paciente decidiu procurar uma segunda opinião médica.

Ao exame oftalmológico, a acuidade visual medida foi movimentos de mão (MM) em olho direito (OD) e 6/6 em olho esquerdo (OE) sem correção. A biomicroscopia do segmento anterior revelou em OD uma massa tumoral localizada às 4 horas, com dimensões de 4,6 $\mathrm{mm}$ antero-posterior, 5,2 $\mathrm{mm}$ circunferencialmente, e espessura estimada em 2,5 a 2,75 mm obstruindo o ângulo da câmara anterior. A tumoração apresentava coloração esbranquiçada, superfície irregular e proeminente vascularização (Figura 2). OE apresentava-se dentro dos limites da normalidade. A pressão intra-ocular (PO) medida com tonômetro de Perkins foi $18 \mathrm{mmHg}$ OD e $12 \mathrm{mmHg}$ OE. A oftalmoscopia indireta sob midríase medicamentosa não apresentou alterações em ambos os olhos. A ecografia convencional (modo A e B) com sonda de $15 \mathrm{mHz}$ (Mentor Ophthalmic, Norwell, MA) não revelou a margem posterior do tumor.

Outros exames complementares como tomografia computadorizada de crânio e abdome, raio $\mathrm{X}$ de tórax não revelaram quaisquer anormalidades. A avaliação oncológica sistêmica foi negativa.

A hipótese inicial foi tratar-se de um melanoma amelanóti-

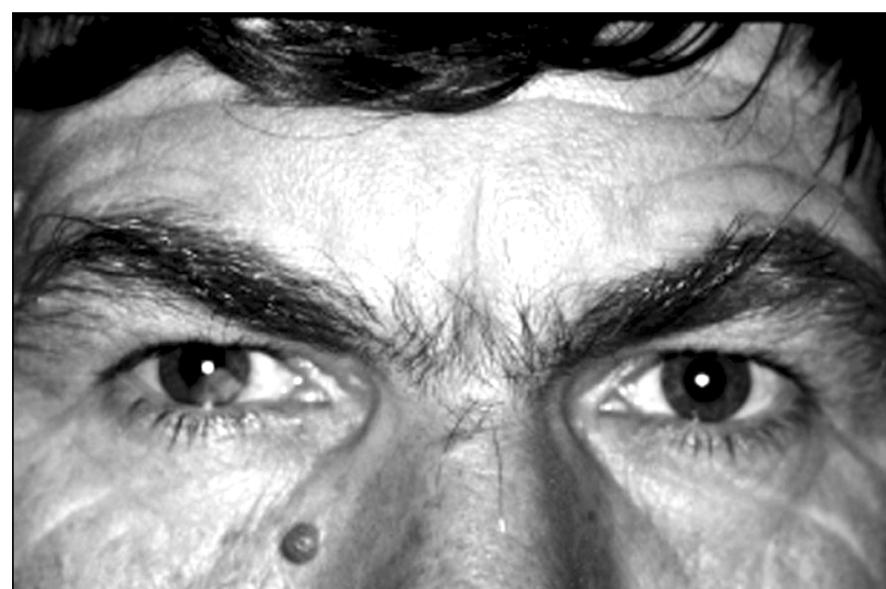

Figura 1 - Fotografia frontal do paciente apresentando lesão iriana suspeita OD. Nota-se a presença de massa na câmara anterior invadindo o eixo visual, nesta imagem a pupila está sob midríase medicamentosa

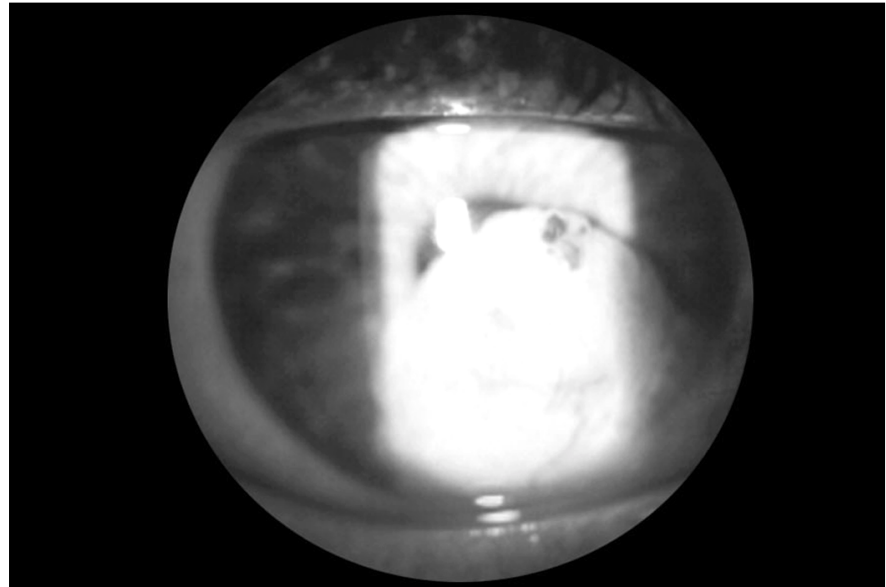

Figura 2 - Fotografia com detalhe do globo ocular direito contendo massa suspeita de melanoma amelanótico ou carcinoma metastático de íris

co de íris ou metástase intra-ocular. Por ainda ter visão neste olho ( $\mathrm{AV}=\mathrm{MM})$, não apresentar aumento da $\mathrm{PO}$, ter sido contra-indicado tratamento da lesão por outro oftalmologista e não ter história pregressa de outra neoplasia, o paciente recusou qualquer tratamento cirúrgico. Optou-se por realizar biópsia aspirativa com agulha fina (FNAB) com visualização direta de acordo com a técnica descrita por Augsburger (1985) ${ }^{(4)}$.

A biópsia aspirativa com agulha fina foi realizada sob anestesia regional do globo ocular com sedação em ambiente cirúrgico. Após a colocação do blefarostato, foram separados os músculos retos verticais e o reto lateral para imobilizar totalmente o globo ocular. Em seguida foi colocado o microscópio cirúrgico para a visualização em maior detalhe do segmento anterior. A punção foi realizada pela córnea, a $1 \mathrm{~mm}$ do limbo na posição das 10 horas. A agulha usada $(25 \times 7)$ foi conectada a um tubo de silicone com com cerca de $30 \mathrm{~cm}$ de extensão, e este conectado à seringa de $10 \mathrm{ml}$ que ficou nas mãos do auxiliar. A agulha introduzida pela córnea foi então direcionada para o tumor e este perfurado para colheita de material. Após a introdução do bisel da agulha dentro do tumor, o auxiliar realizou aspiração manual procurando não desestabilizar a mão do cirurgião. A agulha foi retirada em seguida e o auxiliar fez uma suave compressão sobre o local puncionado durante cerca de 40 segundos para evitar hipotonia. Depois foram aspirados cerca de 2 a $3 \mathrm{ml}$ de BSS através da agulha e tubo usados na biópsia para coletar todo o material dentro da seringa.

O mesmo procedimento foi repetido, usando a mesma punção de entrada para colher células de outra parte do tumor. O material obtido nas duas colheitas foi encaminhado para a análise citológica.

Durante a segunda aspiração da biópsia ocorreu sangramento e hifema total da câmara anterior. Apesar disto, o paciente evoluiu bem, não apresentou aumento da $\mathrm{PO}$ após o procedimento e houve total reabsorção do hifema em cerca de 15 dias. No primeiro exame da citologia, o resultado foi negati- 
vo para células neoplásicas. Foi solicitada uma nova avaliação da lâmina pelo Depto. de Patologia do Wills Eye Hospital* que detectou a presença de células malignas características de melanoma amelanótico de íris tipo misto (fusiforme e epitelióide) (Figura 3). Devido ao tamanho da lesão e à alta malignidade do melanoma do tipo misto, o patologista do Wills Eye Hospital recomendou a enucleação do globo ocular. O paciente concordou com o procedimento e, após consentimento escrito, foi realizada cirurgia. $\mathrm{O}$ anátomo-patológico do olho enucleado confirmou o diagnóstico citológico de melanoma maligno primário de íris do tipo misto e mostrou a invasão para corpo ciliar e coróide anterior (Figura 4).

O paciente está em acompanhamento oncológico e oftalmológico trimestral, com sobrevida superior a 2 anos, sem sinais de metástases até o momento.

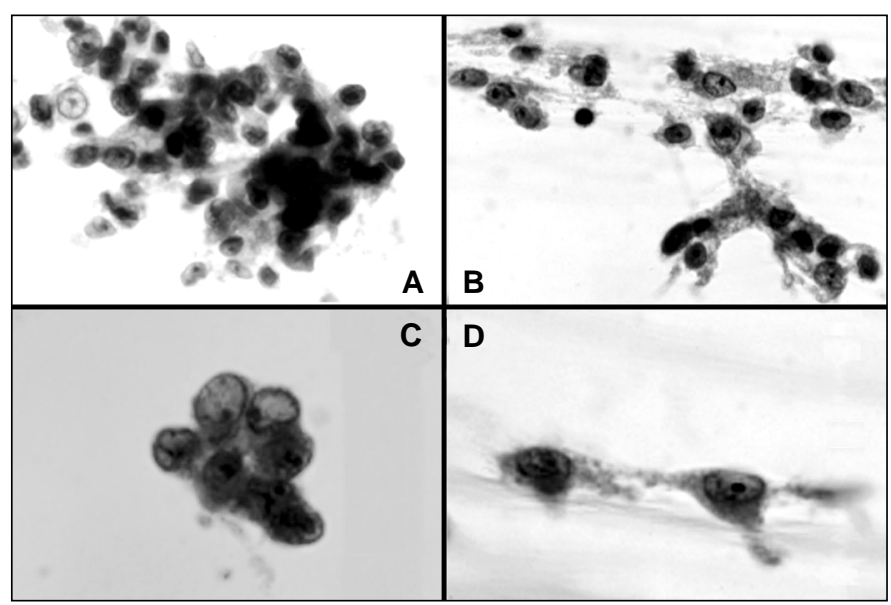

Figura 3 - A e B: Material celular obtido durante FNAB, compatível com melanoma de íris, amostras 1 e 2 respectivamente. C: Células epitelióides com núcleo grande, redondo ou oval, nucléolos proeminentes e margens citoplasmáticas distintas. D: Células com características fusiformes, do tipo B com nucléolo evidente

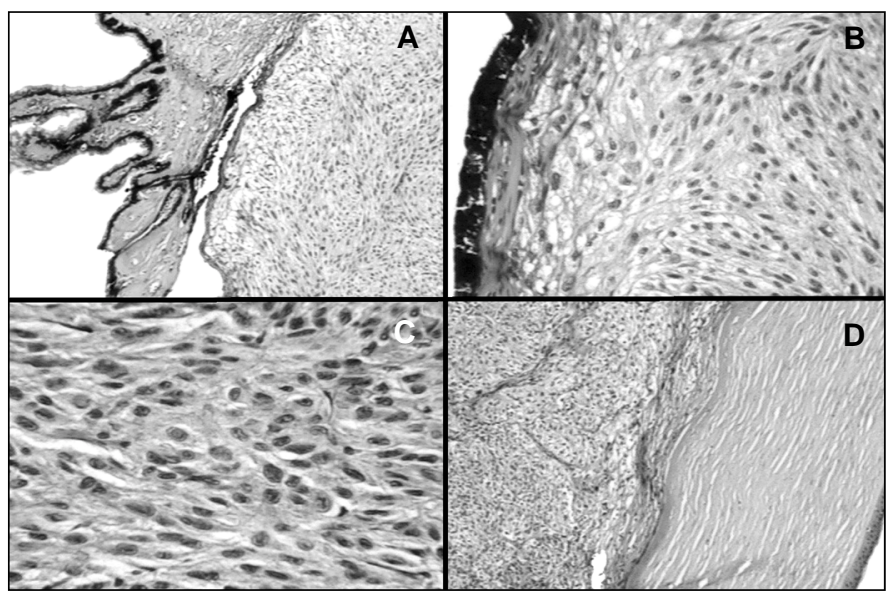

Figura 4 - Cortes histológicos corados com HE do globo ocular enucleado. A: Corpo ciliar acometido pelo tumor. B: Íris com tecido tumoral. C: Imagem em aumento 10x mostra células fusiformes tipo B

e epitelióides. D: Massa tumoral tocando o endotélio da córnea

\begin{tabular}{l} 
RESULTADOS \\
\hline
\end{tabular}

FNAB: Laboratório em Porto Alegre - Negativo para células neoplásicas.

Serviço de Patologia Wills Eye Hospital - Agregados de células tumorais consistentes com melanoma de íris. Algumas células com configuração fusiforme e outras epitelióides, com margens citoplasmáticas distintas. Todas as células contém nucléolo. As células fusiformes são compatíveis com melanoma fusiforme tipo B. As células epitelióides apresentam núcleo grande, redondo ou oval e nucléolo proeminente. Diagnóstico: FNAB consistente com melanoma maligno primário de íris, tipo células mistas (Figura 3).

Anatomopatológico: (Macroscópico) globo ocular medindo 2,2 cm de diâmetro, e 1,2 $\mathrm{cm}$ de coto de nervo óptico. Externamente e ao corte, apresenta na câmara anterior, junto à íris, lesão nodular pardo-clara, medindo $1 \mathrm{~cm}$ no maior diâmetro, invadindo corpo ciliar e coróide anterior. (Microscópico) Melanoma maligno primário de íris, composto por células fusiformes tipo B e algumas células epitelióides. Não há sinais de semeadura tumoral na câmara anterior. O tumor está restrito ao interior do globo ocular (Figura 4).

\section{DISCUSSÃO}

A biópsia aspirativa com agulha fina (FNAB) em oftalmologia é utilizada para colher pequenas amostras de células em lesões intra-oculares suspeitas de neoplasia, bem como lesões inflamatórias com ou sem exsudação ${ }^{(4)}$. Entretanto a indicação deste procedimento deve ser precisa e os riscos devem ser expostos para o paciente de forma bem clara. Neste caso haviam 2 indicações para biópsia: a incerteza diagnóstica devido às características amelanóticas da lesão, que poderia ser um tumor metastático, e a recusa do paciente ao tratamento por não estar completamente convencido do diagnóstico.

Além disto, a experiência do oftalmologista é essencial. Antes de realizar este tipo de procedimento deve-se exercitar a técnica várias vezes, de preferência em olhos enucleados devido a neoplasia, para que o oftalmologista possa realmente colher um aspirado que será analisado no laboratório. Esta segunda parte serve como treino para o patologista que fará a citologia do fluido aspirado ${ }^{(4)}$.

Estudos recentes mostram que a maioria dos tumores de características benignas são coesos o que dificulta a obtenção da $\operatorname{amostra}^{(6)}$. Portanto presume-se que lesões malignas são mais fáceis para se obter uma amostra celular suficiente para o diagnóstico citológico. No caso descrito, apesar do procedimento ter sido bem sucedido, o laboratório de patologia local não era experiente na avaliação citológica de material ocular tão escasso. Diante disto, foi necessário consultar um serviço mais experiente que elucidou o diagnóstico. Esta situação confirma a necessidade de experiência do patologista no processamento e análise deste tipo de material. 
Ademais, o diagnóstico citológico foi essencial na decisão terapêutica e na obtenção do consentimento do paciente para tratar o tumor.

\section{CONCLUSÃO}

A biópsia aspirativa com agulha fina (FNAB), apesar de sua complexidade técnica, é um método confiável no diagnóstico de tumores irianos suspeitos de malignidade.

\section{AGRADECIMENTOS}

Raph C. Eagle Jr., MD - Chefe de Departamento de Patologia do Wills Eye Hospital, pela consulta via correio, fotos da citologia e seu estímulo científico.

Dra. Ana Maria Gaiger e Dra. Luciane Dreher Irion Palologistas Reunidos de Porto Alegre, pelo anatomo-patológico e fotos, além da sua constante colaboração.

\section{ABSTRACT}

To report the case of a patient who presented with a solid anterior segment intraocular mass in his right eye, and the diagnostic investigation chosen by the authors. Diagnostic uncertainty and patient's refusal to agree with the treatment caused the authors to perform a fine needle aspiration biopsy of the lesion. Biopsy was performed by corneal puncture, through the aqueous and the tumor. Cytology of the specimen obtained by the biopsy determined the therapeutic strategy for this case. Cytology diagnosis was consistent with primary malignant melanoma of the choroid of mixed cell type. Enucleation of the eye was suggested due to the position of the tumor and its potential to spread distant metastases. After enucleation, anatomopathological examination of the eye presented a similar result to cytology. The patient is currently been followed, with no signs of metastasis 2 years after enucleation.

Keywords: Choroid neoplasms/pathology; Melanoma/pathology; Needle biopsy; Eye enucleation

\section{REFERÊNCIAS}

1. Martin HE, Ellis EB. Biopsy by needle puncture and aspiration. Ann Surg 1930;92:169-81.

2. Rosai J. Introduction. In: Rosai J editor. Ackerman's surgical pathology. St Louis: Mosby; 1996. p. 1-12.

3. Veasey Jr. CA. Intraocular biopsy. Am J Ophthalmol 1951;34:432-4.

4. Augsburger JJ, Shields JA, Folberg R, Lang W, O'Hara B, Claricci JD. Fine needle aspiration biopsy in the diagnosis of intraocular cancer. Cytologichistologic correlations. Ophthalmology 1985;92:39-49.

5. Augsburger JJ. Fine needle aspiration biopsy in the diagnosis of suspected intraocular cancer. In: Sigelman J, Jakobiec FA editors. Advanced techniques in ocular surgery. Philadelphia: W. B. Saunders; 1984. p. 341-58.

6. Foulds WS. The uses and limitations of intraocular biopsy. Eye 1992;6 (Pt1):11-27.

7. Schneider S, Augsburger JJ, Ehya H. Fine Needle Aspiration Biopsy of solid intraocular tumors: the implications of an insufficient aspirate for cytopathologic diagnosis[abstract]. Invest Ophthalmol Vis Sci 1999;40:S224.

8. Augsburger JJ, Schneider S, Ehya H. The role of transvitreal FNAB in uspected small choroidal melanomas [abstract]. Invest Ophthalmol Vis Sci 1999;40:S224

\title{
XXXVII Reunião Anual do Centro de Estudos Oftalmológicos "Cyro de Rezende"
}

\author{
27 e 28 de Outubro de 2001 \\ Ribeirão Preto - SP
}

INFORMAÇÕES: Fax: $(00 \times x 16) 602-2860$ 